\title{
Carbon nanotubes as a new material for the purification of alcohol-containing liquids
}

\author{
I. Zaporotskova*, N.Polikarpova, T. Ermakova, D. Polikarpov \\ Volgograd State University, Russia
}

Ethyl alcohol, which has a wide application in pharmaceutics, chemical, electronic and food-processing industry, practically always contains various impurities. Many of these impurities are found in quantities which are not sufficient to influence organoleptic effects and toxic potential of ethyl alcohol. However, the quantity of some of them, such as aldehydes, fusel oil, ethers and methyl alcohol is strictly regulated. The paper suggests a new effective method of purifying alcohol-containing liquids from undesirable impurities. At present, development in many areas of science and technology is associated with carbon nanotubes (CNTs) [1-6]. One of their remarkable features of is related to their unique sorption characteristics. The highly curved surface of CNTs can adsorb rather complex molecules including molecules of organic origin. The effectiveness of nanotubes with respect to organic molecules is ten times greater than the that one of graphite adsorbents, which are currently considered the most common means of purification. Therefore, nanotubes can be used for final purification of various liquids from extremely low concentrations of impurities. We performed quantumchemical study of molecul adsorption of heavy alcohols (normal and isomeric propanol) on the outer surface of single-walled carbon nanotubes. We constructed energy curves of interaction of normal propanol and isopropanol molecules with the CNT's surface $(6,6)$. We also established the most effective adsorption centres for molecules of heavy alcohols. We also proved the feasibility of physical adsorption. We developed and implemented a method of alcohol liquids purification from impurities with CNTs. We carried out experimental study of water-ethanol mixtures taken before and after purification with carbon nanomaterials, applying molecular methods of IR spectroscopy, chromatography and chemical analysis and proved the positive effect of carbon nanotubes on the process of purifying alcohol liquids from adverse impurities.

\section{NOTES}

\section{Effect of buckypaper properties on attenuation of electromagnetic radiation}

\author{
F. Komarov, R. Krivosheev, E. Munoz, V. Rodionova, G. Ismailova, I. Parchomova \\ Belarusian State University, Belarus
}

Two different procedures of carbon nanotube foils fabrication are discussed. The size, structural and optical characteristics as well as electrophysical properties of initial nanotubes and prepared buckypaper are studied. The parameters of electromagnetic radiation-buckypaper interaction are derived for the range $8.15-73.3$ $\mathrm{GHz}$. Strong reflection and attenuation of the radiation up to $40 \mathrm{~dB}$ are observed. Such results render this material promising for protection against electromagnetic radiation in sensitive devices and systems, especially in those experiencing elevated mechanical and thermal loads.

NOTES 